\title{
The NeBoP score - a clinical prediction test for evaluation of children with Lyme Neuroborreliosis in Europe
}

\author{
Barbro H. Skogman ${ }^{1,2^{*}}$, Johanna Sjöwall ${ }^{3}$ and Per-Eric Lindgren ${ }^{4,5}$
}

\begin{abstract}
Background: The diagnosis of Lyme neuroborreliosis (LNB) in Europe is based on clinical symptoms and laboratory data, such as pleocytosis and anti-Borrelia antibodies in serum and CSF according to guidelines. However, the decision to start antibiotic treatment on admission cannot be based on Borrelia serology since results are not available at the time of lumbar puncture. Therefore, an early prediction test would be useful in clinical practice. The aim of the study was to develop and evaluate a clinical prediction test for children with LNB in a relevant European setting.

Method: Clinical and laboratory data were collected retrospectively from a cohort of children being evaluated for LNB in Southeast Sweden. A clinical neuroborreliosis prediction test, the NeBoP score, was designed to differentiate between a high and a low risk of having LNB. The NeBoP score was then prospectively validated in a cohort of children being evaluated for LNB in Central and Southeast Sweden $(n=190)$ and controls with other specific diagnoses $(n=49)$.

Results: The sensitivity of the NeBoP score was $90 \%$ (Cl $95 \%$; 82-99 \%) and the specificity was $90 \%$ (Cl $95 \%$; 85-96\%). Thus, the diagnostic accuracy (i.e. how the test correctly discriminates patients from controls) was $90 \%$ and the area under the curve in a ROC analysis was 0.95. The positive predictive value (PPV) was 0.83 (Cl $95 \%$; 0.75-0.93) and the negative predictive value (NPV) was 0.95 (Cl $95 \%$; 0.90-0.99).

Conclusion: The overall diagnostic performance of the NeBoP score is high (90\%) and the test is suggested to be useful for decision-making about early antibiotic treatment in children being evaluated for LNB in European Lyme endemic areas.
\end{abstract}

Keywords: Lyme neuroborreliosis, Lyme borreliosis, Predictive test, Diagnostic accuracy, Children

\section{Background}

Lyme Borreliosis (LB) is caused by the spirochete Borrelia burgdorferi and is the most common tick-borne infection both in Europe and Northern America [1, 2]. The infection may give rise to different symptoms by affecting organs such as the skin, joints, heart muscle or nervous system [3-5]. The diagnosis of Lyme neuroborreliosis (LNB) in Europe is based on clinical symptoms and laboratory findings, including pleocytosis in the

\footnotetext{
* Correspondence: barbro.hedinskogman@ltdalarna.se

'Paediatric clinic, Falun General Hospital, Nissers väg 3, S-791 82 Falun, Sweden

${ }^{2}$ Center for Clinical Research (CKF) Dalarna-Uppsala University, S-791 31

Falun, Sweden

Full list of author information is available at the end of the article
}

cerebrospinal fluid (CSF) and intrathecal anti-Borrelia antibody production, in accordance with the guidelines [6]. However, the decision to start antibiotic treatment on admission cannot be based on anti-Borrelia antibodies in CSF, since test results are not available at the time of lumbar puncture. A prediction test would therefore be useful in clinical practice for decision-making about early start of antibiotic treatment.

Previous studies have suggested different clinical prediction rules but patients have not been representative of children with LNB in Europe [7-10]. Studies on large representative samples of all children being evaluated for LNB in European Lyme endemic areas are warranted. 
Facial nerve palsy is the most common neurological finding among children with LNB in Europe [11, 12], but unspecific symptoms such as fatigue, low-grade fever, nausea and loss of appetite may often occur, without being accompanied with specific neurological findings [13]. The clinical picture in children with LNB is similar in Central and Northern Europe [14, 15] where the tick vector Ixodes ricinus is dominant and Borrelia burgdoferi sensu lato $(B b)$ is present in mainly three humanpathogenic species; B. afzelii, B. garinii and $B b$ senso stricto [1]. In Northern America, there are several different tick vectors and the main human pathogenic species is $B b$ senso stricto [2]. It is well known that the clinical picture of LNB in childhood differs in Europe compared to Northern America, where facial nerve palsy is less frequent but EM in combination with neurological symptoms occur more often [16-18]. Consequently, to find a common clinical predictive test valid for paediatric LNB patients on both continents is not feasible.

The aim of the study was to develop and evaluate a clinical prediction test for children with LNB in a relevant European setting.

\section{Methods}

Development of a clinical prediction test-the NeBoP score Clinical and laboratory data was collected retrospectively from a large cohort of well-characterized and representative children being evaluated for LNB in Southeast Sweden during the period $(2000-2005)(n=177)$ [12]. This cohort was used for development and evaluation of the NeBoP score. Data was analysed in a logistic regression model to find independent and statistically significant variables to discriminate between "Confirmed LNB" and "Not determined". Patients in "Confirmed LNB" were classified as "Definite LNB" patients according to European guidelines at the time [1], i.e. the same criteria as now [6]. Patients in "Not determined" were similar to "Non LNB", i.e. patients with acute idiopathic facial nerve palsy, tension headache and patients with unspecific symptoms without LNB diagnosis [12]. Variables such as age, gender, headache, durations of symptoms, known tick bite or time of the year on admission did not differ between groups. Significant variables that came out in the logistic regression model were: 1) acute facial nerve palsy, 2) fever $38-39^{\circ} \mathrm{C}$, 3) fatigue, 4) erythema migrans and/or lymphocytoma, 5) pleocytosis in CSF (with total cell count $\geq 5 \times 10^{6} / \mathrm{L}$ with $\geq 90 \%$ mononuclear cells).

Out of these five significant variables, the NeBoP score was designed, including weighed points (p) to differentiate between high and low probability of having LNB (Fig. 1). Definitions and instructions to the paediatrician were added to ensure equal and correct interpretation of the patient's symptoms (Fig. 1). The NeBoP score was also pretested on a small group of paediatricians and minor corrections were made to ensure validity.

\section{Evaluation of cut-off levels for the NeBoP score}

The performance of the NeBoP score at different cut-off levels was evaluated on data from the retrospective patient material [12] and shown in Table 1. A high sensitivity $(100 \%)$ at the best positive predictive value $(0.60)$ was considered preferential, and the cut-off level was set at 3 points for a positive NeBoP score (Fig. 1 and Table 1).

\section{Patient sample for validation of the NeBoP score}

During the years 2010-2013, 197 children were evaluated for LNB at seven paediatric clinics in Central and Southeast Sweden. Children and parents/guardians were asked to participate in the study at the admission stage, and patients were consecutively enrolled as a prospective cohort. This cohort is considered representative for all paediatric patients being evaluated for LNB in a relevant European clinical setting, and is therefore suitable for this present study. CSF and blood samples were taken on admission for laboratory evaluation and the child and parents/ guardians completed a standardized questionnaire. The paediatrician, following preset instructions and definitions, completed the NeBoP score for each patient on admission, before anti-Borrelia antibody results were available and before the patient was diagnosed as LNB or non-LNB. A two-month evaluation of clinical outcome was part of the study, but no serum samples were taken at follow-up.

Out of 197 patients included in the study, seven children were excluded because of missing data. These children $(n=7)$ did not differ in age or gender from patients included in the study $(n=190)$.

\section{Control sample for validation of the NeBoP score}

Children being evaluated and diagnosed with other specific diagnoses during the study period were asked together with parents/guardians to participate in the study and were consecutively enrolled as controls $(n=49)$. Patients were not included from all seven paediatric clinics so this control sample cannot be considered as representative of children with each diagnosis. Instead, they represent a diversity of patients with other infectious, immunological and neurological diseases. Controls were children with enteroviral meningitis $(n=7)$, unspecified viral meningitis $(n=6)$, tick-borne encephalitis $(n=3)$, varicella zoster $(n=1)$, mycoplasma infection with neurological symptoms but negative PCR in CSF $(n=2)$, pneumonia with headache and normal CSF $(n=1)$, postinfectious encephalitis $(n=3)$, periodic fever $(n=1)$, unspecified autoimmune disease $(n=1)$, polyneuropathy $(n=1)$, Guillain-Barre syndrome $(n=1)$, multiple 


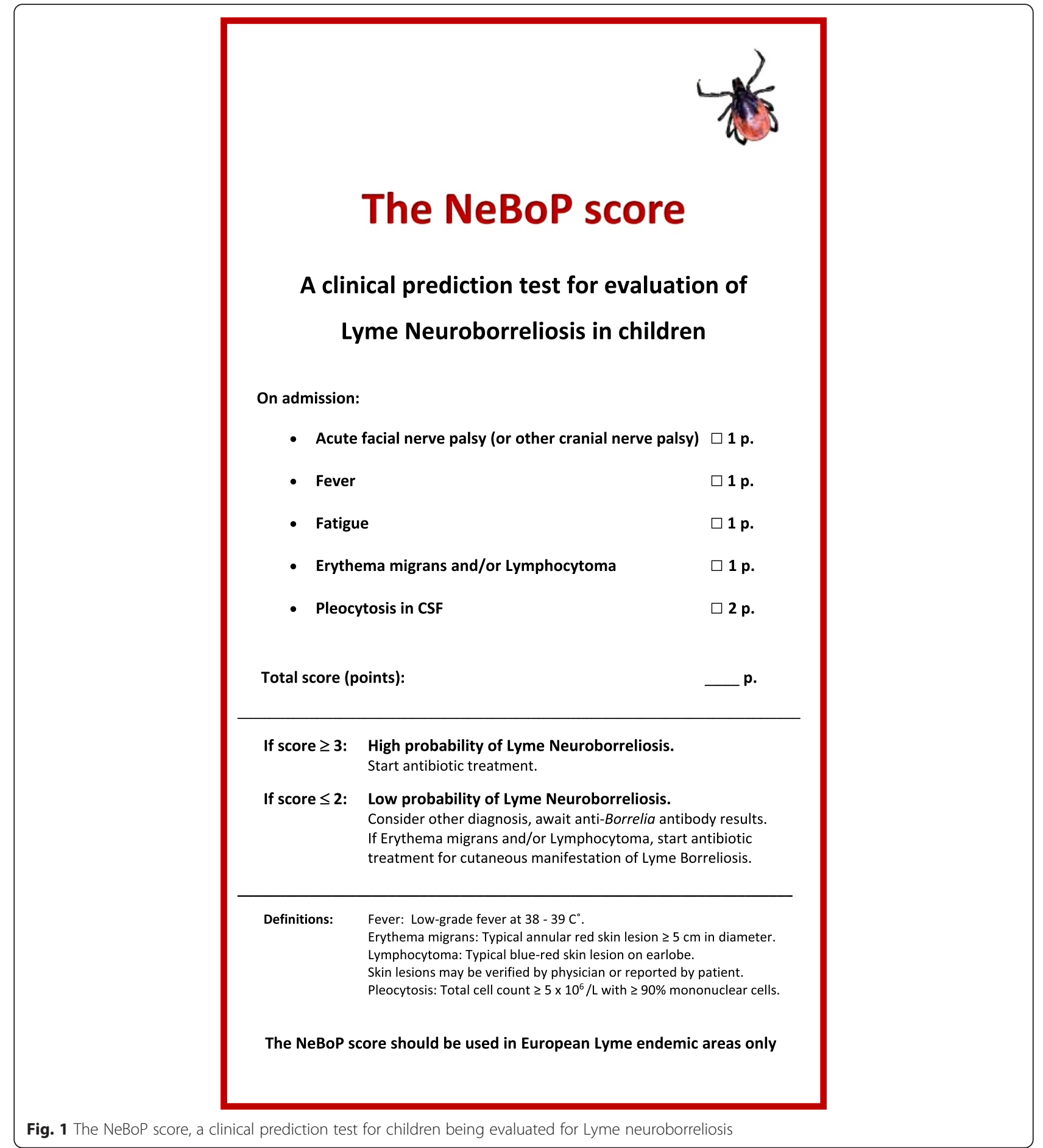

sclerosis $(n=1)$, myasthenia gravis $(n=1)$, narcolepsy $(n$ $=1)$, neurofibromatosis type $1(n=1)$, ischemic stroke $(n=1)$, febrile seizure $(n=1)$, infantile spasm $(n=3)$, epilepsy $(n=2)$, idiopathic intracranial hypertension $(n=2)$, migraine headache $(n=3)$, tension headache $(n=3)$, head trauma $(n=3)$.

\section{Classification of patients and controls}

According to European guidelines, classification of patients as "Definite LNB" and "Possible LNB" was based on neurological symptoms indicative for LNB and laboratory findings in CSF. [6] Patients who did not meet the criteria for either of the two groups were classified 
Table 1 Cut-off levels for the NeBoP score

\begin{tabular}{lllll}
\hline Cut-off & Sensitivity (\%) & Specificity (\%) & PPV $^{\#}$ & NPV $^{*}$ \\
\hline Score $\geq 2$ p & 100 & 42 & 0.54 & 1.00 \\
Score $\geq 3$ p & 100 & 54 & 0.60 & 1.00 \\
Score $\geq 4$ p & 78 & 64 & 0.60 & 0.82 \\
Score $\geq 5$ p & 25 & 85 & 0.53 & 0.62
\end{tabular}

\# $\mathrm{PPV}=$ positive predictive value

${ }^{*} \mathrm{NPV}=$ negative predictive value

$p=$ score points $(1-6 p)$

Calculations are based on a retrospective cohort with "Confirmed LNB" as patients and "Not determined" as controls (12)

as "Non-LNB" and patients with other specific diagnoses were classified as "Controls" (Table 2).

Pleocytosis in CSF was defined as total cell count $\geq 5 \times$ $10^{6} / \mathrm{L}$ [19-21]. Intrathecal anti-Borrelia antibody production (IgG and/or IgM) was analysed with the routine assay IDEIA Lyme neuroborreliosis kit according to manufacturer's instructions (Oxoid, Hampshire, UK) [22].

\section{Characteristics of patients}

Clinical characteristics and laboratory data from patients being evaluated for LNB $(n=190)$ are shown in Table 3. Headache, fatigue, facial nerve palsy and loss of appetite were major clinical manifestations and known tick bite was reported from $53 \%$ of patients. Ninety-nine patients $(n=99)$ received antibiotic treatment. Patients were diagnosed as "Definite LNB" $(n=52)$, "Possible LNB" $(n=31)$ and "Non-LNB" ( $n=107)$ according to guidelines (Table 3) [6]. Patients in the "Non-LNB" were patients with acute idiopathic facial nerve palsy, tension headache and patients with unspecific symptom without LNB diagnosis.

Table 2 Classification of children being evaluated for Lyme neuroborreliosis and controls

\begin{tabular}{|c|c|}
\hline Diagnosis & Criteria \\
\hline \multirow[t]{3}{*}{ Definite LNB ${ }^{\S}$} & $\begin{array}{l}\text { 1. Neurological symptoms indicative for LNB without } \\
\text { other obvious reasons }\end{array}$ \\
\hline & 2. Pleocytosis in CSF \\
\hline & $\begin{array}{l}\text { 3. Intrathecal anti-Borrelia antibody production } \\
(\lg G \text { and/or } \lg M)^{\#}\end{array}$ \\
\hline Possible LNB ${ }^{\S}$ & Two of the criteria for Definite LNB are fullfilled \\
\hline Non-LNB & $\begin{array}{l}\text { Not meeting the criteria for Definite LNB or Possible } \\
\text { LNB }\end{array}$ \\
\hline Controls & Other specific diagnosis \\
\hline
\end{tabular}

Total cell count $\geq 5 \times 10^{6} / \mathrm{L}$ in CSF

${ }^{\S}$ Classified according to European guidelines (6)

" Detected by IDEIA Lyme neuroborreliosis assay (22)

LNB $=$ Lyme neuroborreliosis

$\mathrm{CSF}=$ cerebrospinal fluid

$\mathrm{Ig}=\mathrm{Immunoglobulin}$
Table 3 Characteristics of children being evaluated for Lyme neuroborreliosis

\begin{tabular}{|c|c|}
\hline On admission & Patients $(n=190)$ \\
\hline Age, median years (range) & $10(1-19)$ \\
\hline \multicolumn{2}{|l|}{ Sex } \\
\hline female, n (\%) & $105(55)$ \\
\hline male, n (\%) & $85(45)$ \\
\hline Known tick bite, n (\%) & $100(53)$ \\
\hline \multicolumn{2}{|l|}{ Major clinical features } \\
\hline Acute facial nerve palsy, $\mathrm{n}(\%)$ & $93(49)$ \\
\hline Headache, n (\%) & $136(72)$ \\
\hline Fatigue, n (\%) & $144(76)$ \\
\hline Fever, n (\%) & $59(31)$ \\
\hline Neck pain, n (\%) & $71(37)$ \\
\hline Neck stiffness, n (\%) & $44(23)$ \\
\hline Loss of appetite, n (\%) & $89(47)$ \\
\hline Nausea, n (\%) & $68(36)$ \\
\hline Vertigo, n (\%) & $63(33)$ \\
\hline Radiating pain, n (\%) & $29(15)$ \\
\hline Erythema migrans (EM) and/or lymphocytoma, n (\%) & $42(22)$ \\
\hline \multicolumn{2}{|l|}{ Laboratory findings } \\
\hline Pleocytosis in CSF, n (\%) & $82(43)$ \\
\hline with $\geq 90 \%$ mononuclear cells, n (\%) & $75(91)$ \\
\hline Pleocytosis in CSF, median (range) & $142(8-890)$ \\
\hline Anti-Borrelia antibodies in CSF, $n(\%){ }^{\#}$ & $53(28)$ \\
\hline $\lg M, n(\%)$ & $9(5)$ \\
\hline $\lg G, n(\%)$ & $12(6)$ \\
\hline $\lg M+\lg G, n(\%)$ & $32(17)$ \\
\hline Anti-Borrelia antibodies in serum, $n$ (\%) & $83(44)$ \\
\hline $\lg M, n(\%)$ & $22(12)$ \\
\hline $\operatorname{lgG}, \mathrm{n}(\%)$ & $26(14)$ \\
\hline $\lg M+\lg G, n(\%)$ & $35(18)$ \\
\hline Antibiotic treatment, n (\%) & $99(52)$ \\
\hline \multicolumn{2}{|l|}{ Diagnosis $\S$} \\
\hline Definite LNB, n (\%) & $52(27)$ \\
\hline Possible LNB, n (\%) & $31(16)$ \\
\hline Non-LNB, n (\%) & $107(56)$ \\
\hline
\end{tabular}

Total cell count $\geq 5 \times 10^{6} / \mathrm{L}$ cells in CSF

" Detected by IDEIA Lyme neuroborreliosis assay (22)

${ }^{5}$ Classified according to European guidelines (6)

$\mathrm{CSF}=$ Cerebrospinal fluid

$\mathrm{Ig}=$ Immunoglobulin

LNB $=$ Lyme neuroborrelios

\section{Characteristics of controls}

Characteristics of children with other specific diagnoses $(n=49)$ are shown in Table 4. All controls were negative to anti-Borrelia antibodies in CSF but four children $(n=4)$ had anti-Borrelia IgG antibodies in 
Table 4 Characteristics of children with other specific diagnosis (controls)

\begin{tabular}{ll}
\hline On admission & Controls $(n=49)$ \\
\hline Age, median years (range) & $10(0-19)$ \\
Sex & \\
Female, $\mathrm{n}(\%)$ & $26(53)$ \\
Male, $\mathrm{n}(\%)$ & $23(47)$ \\
Known tick bite, $\mathrm{n}(\%)$ & $12(24)$ \\
Laboratory findings & \\
Pleocytosis in CSF, $\mathrm{n}(\%)$ & $15(31)$ \\
$\quad$ with $\geq 90 \%$ mononuclear cells, $\mathrm{n}(\%)$ & $4(27)$ \\
Pleocytosis in CSF, median (range) & $50(6-1125)$ \\
Anti-Borrelia antibodies in CSF, $\mathrm{n}(\%)$ & $0(0)$ \\
Anti-Borrelia antibodies in serum (lgG), $\mathrm{n}(\%)$ & $4(8)$ \\
Diagnosis & \\
Viral meningitis (enterovirus), $\mathrm{n}(\%)$ & $7(15)$ \\
Viral meningitis (unspecified), $\mathrm{n}(\%)$ & $6(12)$ \\
Tick-borne encephalitis (TBE), $\mathrm{n}(\%)$ & $3(6)$ \\
Other infectious disease, $\mathrm{n}(\%)$ & $4(8)$ \\
Post-infectious encephalitis, $\mathrm{n}(\%)$ & $3(6)$ \\
Other immunological disease, $\mathrm{n}(\%)$ & $2(4)$ \\
Other neurological disease, $\mathrm{n}(\%)$ & $18(37)$ \\
Tension headache, $\mathrm{n}(\%)$ & $3(6)$ \\
Head trauma, $\mathrm{n}$ (\%) & $3(6)$ \\
\hline
\end{tabular}

Total cell count $\geq 5 \times 10^{6} / \mathrm{L}$ in CSF

\# Detected by IDEIA Lyme neuroborreliosis assay (22) $\mathrm{CSF}=$ Cerebrospinal fluid

Ig Immunoglobulin

serum. Known tick bites were reported from $24 \%$ but no child in the control group had received antibiotic treatment for LNB. The different specific diagnoses among controls are described above under control sample.

\section{Questionnaire}

A structured questionnaire was used for data collection on admission and at the two-month follow-up. It consisted of questions to children and parents/guardians concerning current symptoms, known tick bites, previous antibiotic treatment of LB and the basic health of the child (as shown in Additional file 1). Children and parents/guardians in the control group received a similar, but slightly modified, questionnaire. At the twomonth follow-up, questions focused on persistent symptoms and time to recovery.

\section{Statistics}

SPSS software, version 21 and SISA-binomial were used for statistical calculations. A logistic regression was used to find independent and statistically significant variables to discriminate between "Definite LNB" and "Non LNB" in the retrospective cohort as described above. A $p$ value $<0.05$ was considered significant. In the present prospective cohort, the diagnostic accuracy of the NeBoP score was calculated on "Definite LNB" and "Possible LNB" as patients and "Non-LNB" and "Controls" as controls. A receiver operating characteristic (ROC) curve with calculated area under the curve (AUC) was used to illustrate the results (Fig. 2).

\section{Ethics}

Informed written consent was received from all children and parents/guardians included in the study. Approval of the study was obtained from the Regional Ethics Committee in Uppsala, Sweden (Dnr 2010/106).

\section{Results}

\section{Diagnostic performance of the NeBoP score}

Results from the NeBoP score in different diagnostic groups are shown in Table 5 . Among children classified as "Definite LNB", 51 out of 52 (98\%) had a positive $\mathrm{NeBoP}$ score and among children with "Possible LNB", 24 out of $31(77 \%)$ had a positive test. The majority of children in "Non-LNB" and "Controls" had a negative NeBoP score (91\% and $90 \%$ respectively) (Table 5).

The sensitivity of the NeBoP score was $90 \%(95 \% \mathrm{CI}$; 82-99 \%), calculated on patients with "Definite LNB" and "Possible LNB". The specificity of the test was $90 \%$

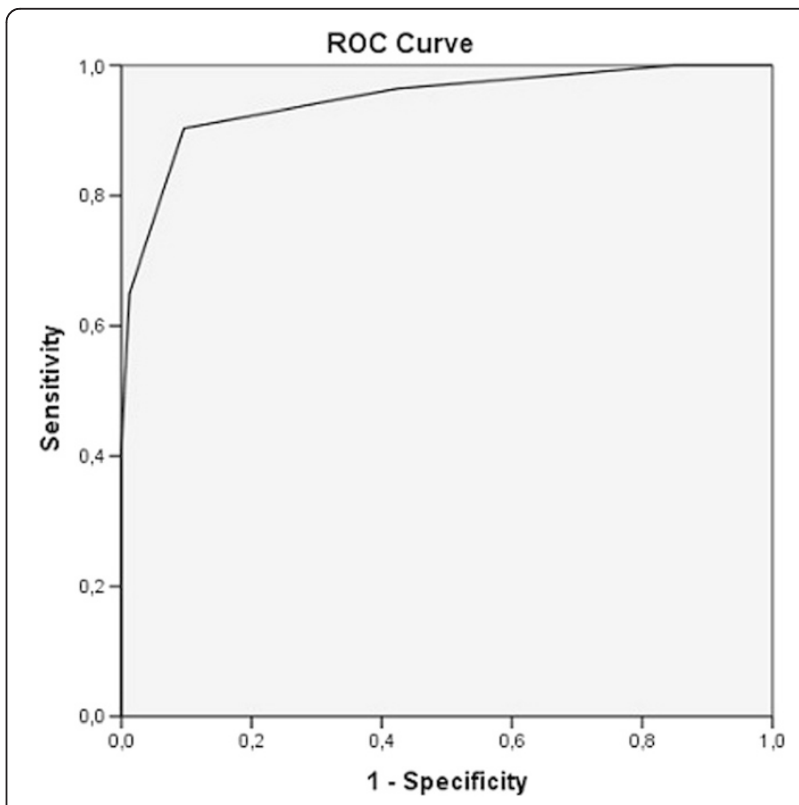

Fig. 2 The diagnostic accuracy of the NeBoP score shown as a ROC curve. The area under the curve (AUC) was $0.95(p<0.0001)$. Calculations are based on "Definite LNB" $(n=52)$ and "Possible $\operatorname{LNB}^{\prime}(n=31)$ as patients and "Non-LNB" $(n=107)$ and "Controls" $(n=$ 49) as controls. ROC curve $=$ Receiver Operator Characteristic curve 
Table 5 Results of the NeBoP score in different diagnostic groups

\begin{tabular}{llll}
\hline Diagnosis & \multicolumn{2}{l}{ NeBoP score } & \\
\cline { 2 - 3 } & Positive & Negative & Total \\
\hline Definite LNB, n (\%) & $51(98)$ & $1(2)$ & 52 \\
Possible LNB, n (\%) & $24(77)$ & $7(23)$ & 31 \\
Non-LNB, n (\%) & $10(9)$ & $97(91)$ & 107 \\
Controls, n (\%) & $5(10)$ & $44(90)$ & 49 \\
\hline
\end{tabular}

LNB = Lyme neuroborreliosis, classified according to European guidelines (6) Positive test $\geq 3$ points, negative test $\leq 2$ points

(95 \% CI; 85-96\%), calculated on patients with "NonLNB" and "Controls" (Table 6). Thus, the overall diagnostic accuracy of the NeBoP score (i.e. how the test correctly defines patients and controls) was $90 \%$. Results are also shown as a ROC curve with an area under the curve (AUC) of 0.95 ( $p<0.001)$ (Fig. 2).

The positive predictive value (PPV) of the NeBoP score was 0.83 (95\% CI; 0.75-0.93) and the negative predictive value (NPV) was 0.95 (95\% CI; 0.90-0.99). Likelihood ratios (LR) are shown in Table 6.

\section{Distribution of clinical symptoms and NeBoP score points}

The distribution of clinical symptoms and NeBoP score points among children being evaluated for LNB $(n=190)$ are shown in Fig. 3. Among patients with $\geq$ $3 \mathrm{p}$ in the NeBoP score, the most common combination of symptoms was facial nerve palsy, low-grade fever and fatigue in combination with pleocytosis in CSF (Fig. 3).

\section{Discussion}

This study shows a high diagnostic accuracy (90\%) of the NeBoP score in children being evaluated for LNB in a Northern European Lyme endemic area. Consequently, our results support that this clinical predictive test could be useful for paediatricians in early assessment of children being evaluated for LNB. The NeBoP score is

Table 6 Diagnostic performance of the NeBoP score

\begin{tabular}{ll}
\hline & NeBoP score \\
\hline Sensitivity, $(95 \% \mathrm{Cl})$ & $90 \%(82-99 \%)$ \\
Specificity, $(95 \% \mathrm{Cl})$ & $90 \%(85-96 \%)$ \\
Positive predictive value (PPV), $(95 \% \mathrm{Cl})$ & $0,83(0.75-0.93)$ \\
Negative predictive value (NPV), (95\% Cl) & $0,95(0.90-0.99)$ \\
Positive likelihood ratio (LR+), (95\% Cl) & $9.34(5.05-17.47)$ \\
Negative likelihood ratio (LR-), (95\% Cl) & $0.11(0.05-0.25)$ \\
\hline
\end{tabular}

Calculations are based on "Definite LNB" $(n=52)$ and "Possible LNB" $(n=31)$ as patients and "Non-LNB" $(n=107)$ and "Controls" $(n=49)$ as controls $\mathrm{Cl}=$ Confidence interval applicable in Lyme endemic areas in Central and Northern Europe since there are known similarities in LNB in childhood $[15,16]$. However, the test is not recommended for Northern America due to differences in the clinical manifestations of LNB, the occurrence of different tick vectors and different Borrelia species between the two continents $[16,17]$.

Admittedly, there are a few patients being incorrectly predicted with using the NeBoP score in the present study, which needs to be addressed. Among children classified as "Definite LNB", $98 \%$ had a positive NeBoP score ( $\geq 3 \mathrm{p}$ ) but one patient had negative test (Table 5). This patient had unilateral abducens palsy with pleocytosis and anti-Borrelia antibodies in CSF, (correctly classified as "Definite LNB") but received only 2 NeBoP score points (negative test). Consequently, the NeBoP score should also include other cranial nerve palsy since LNB patients may present with both abducens-and/or oculomotorius nerve palsy [23]. This detail is added in the final version of the NeBoP score (Fig. 1).

Among children classified as "Possible LNB", the majority (77\%) had positive NeBoP scores whereas seven patients $(23 \%)$ had negative tests (Table 5). Six of these patients presented with short duration of symptoms (1-6 days of headache and/or facial nerve palsy) in combination with pleocytosis in CSF, indicating an early LNB. One patient had anti-Borrelia IgM antibodies in serum, one had anti-Borrelia IgG antibodies and one had both IgM and anti-Borrelia IgG antibodies in serum. All patients responded well to antibiotic treatment. It is uncertain whether these six patients were LNB patients with negative NeBoP score due to an untypical distribution of percentage of mononuclear cells in CSF (40-88 \% of total cell count in CSF) or whether symptoms actually derive from other etiology. Unfortunately, these patients were not tested for other tick-borne infections or enteroviral PCR in CSF. Furthermore, one patient with a negative NeBoP score in the "Possible LNB" group presented with very long duration of symptoms, anti-Borrelia IgG antibodies in CSF but no pleocytosis in CSF. The patient had a history of a previously treated LNB. Findings may indicate persistent symptoms as sequelae after a previous LNB infection (despite adequate antibiotic treatment) or ongoing infection without pleocytosis in CSF. The patient again received antibiotic treatment and symptoms were slightly reduced but did not resolve totally.

Our results show that the sensitivity of the NeBoP score is excellent in "Definite LNB", (98 \%) and acceptable in "Possible LNB" (77 \%), with an overall sensitivity of $90 \%$ for the two groups together. Thus, the NeBoP score will help the paediatrician to decide about early antibiotic treatment before test results of anti-Borrelia antibodies in serum and CSF are available. Regarding the Borrelia serology, one should always keep in mind that 


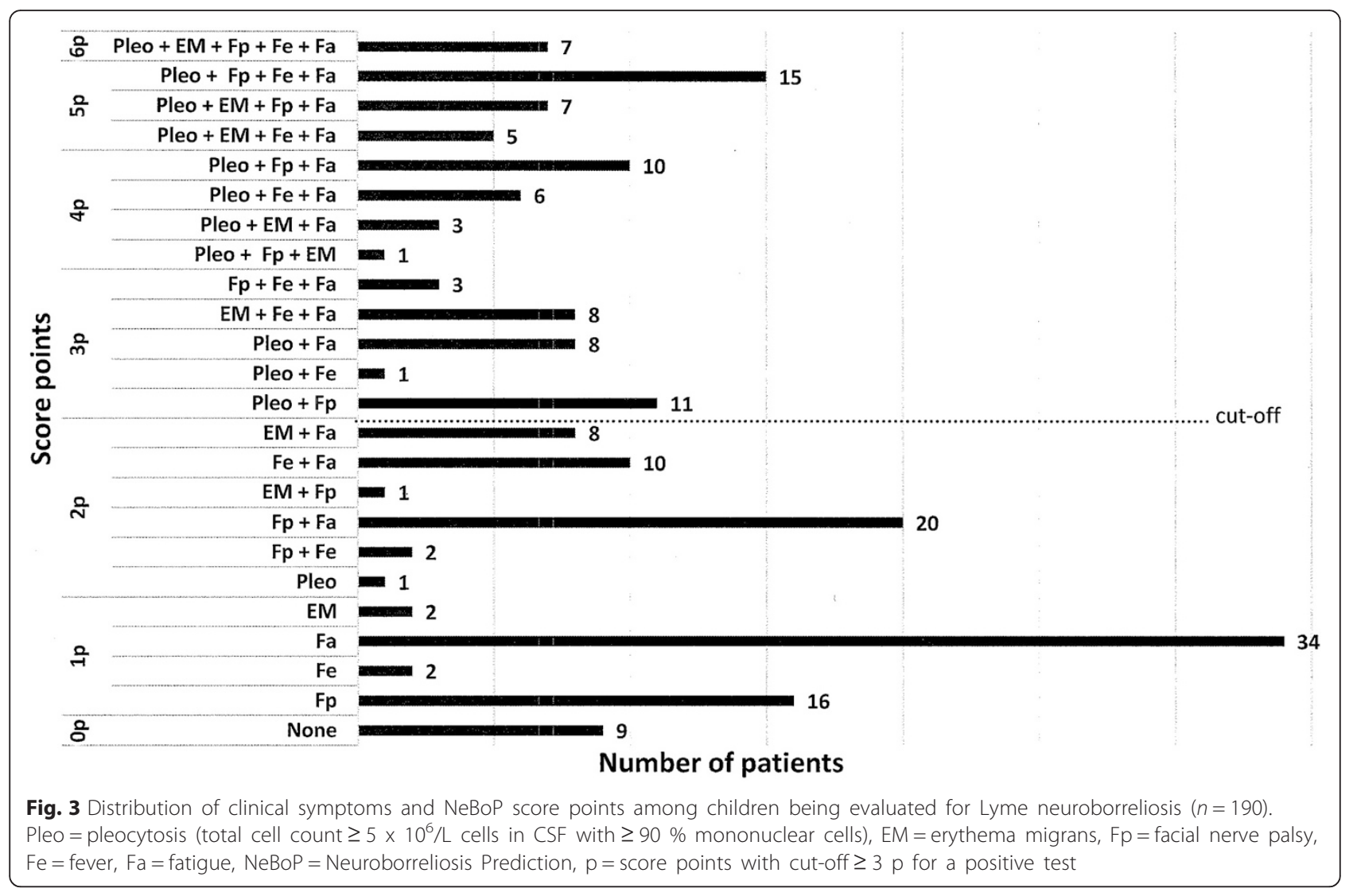

both anti-Borrelia IgG and IgM antibodies in serum should be interpreted with caution because of low sensitivity and specificity [6].

Concerning the two control groups in this study, the heterogeneity of the negative controls $(n=49)$ could be put under further considerations since they represent many different diagnoses without similar clinical manifestations to LNB. However, when evaluating a predictive test it is of importance to include controls without clinical similarity to patients (49 negative controls) as well as controls with clinical similarity to LNB patients from a clinical relevant setting (107 Non-LNB patients). We have included these two control groups in our study and we believe the heterogeneity of controls with other diagnosis therefore can be acceptable. Negative controls without any symptoms (i.e. healthy controls) could not be included in the study due to the fact that a lumbar puncture cannot be performed on healthy children out of ethical reasons.

A control group including a higher number of patients with enteroviral and/or bacterial meningitis should admittedly have been preferable, which is a weakness of the study. Furthermore, the sample size of negative controls could have been larger than 49, but it could unfortunately not be achieve during the time period and in clinical setting of the study. Median age and sex distribution do not differ between patients (Table 3) and controls (Table 4), which is a strength of the study.

In the "Non-LNB" group, $91 \%$ had a negative NeBoP score $(\leq 2 \mathrm{p})$ indicating a high specificity (Table 5$)$. However, 10 children (9\%) in "Non-LNB" scored $\geq 3 \mathrm{p}$ (positive test). Three of these 10 children had acute facial nerve palsy, fever and fatigue with short duration of symptoms, but no pleocytosis or anti-Borrelia antibodies in CSF. These patients may have had idiopathic facial nerve palsy or very early LNB with peripheral cranial nerve palsy but not yet pleocytosis in CSF. Furthermore, seven patients in the "Non LNB" group scored $\geq 3 \mathrm{p}$ (positive test) due to fever, fatigue and EM but had normal CSF, indicating a cutaneous LB with systemic symptoms. These patients are probably incorrectly predicted as LNB by the NeBoP score and should instead be classified as cutaneous LB and receive treatment as such. This instruction is added in the final version of the $\mathrm{NeBoP}$ score (Fig. 1). Again, it should be pointed out how important an evaluation of CSF is, concerning patients with EM and systemic symptoms, since symptoms may indicate an early LNB [24].

Among controls, four children scored $\geq 3 \mathrm{p}$ (positive test) due to viral meningitis with fatigue and pleocytosis with $\geq 90 \%$ mononuclear cells in CSF, yielding a false 
positive NeBoP score. However, these patients had a clinical picture with clear meningeal symptoms in addition to fever and fatigue, making it easy for the paediatrician to clinically distinguish them from patients with Lyme meningitis. Two of them were positive for enterovirus PCR in CSF.

One patient among controls had a periodic fever with fatigue, low-grade fever and a red skin lesion similar to an EM, which resulted in a misclassification by the NeBoP score (3 p). EM is a clinical diagnosis but it is well know that the EM skin lesions may be heterogeneous and may result in misdiagnosis [24]. In such cases, Borrelia serology is not useful due to low sensitivity [24].

In our cohort of children, 15 out of 107 patients in "Non-LNB" received antibiotic treatment on admission before anti-Borrelia antibody results were available. We believe, based on results from our present study with a NPV of 0.95 for the NeBoP score, that the test will be helpful for the paediatrician to correctly refrain from antibiotic treatment on admission and consider other differential diagnoses while waiting for anti-Borrelia antibody results.

Whether the NeBoP score is more helpful as a decision tool for the paediatrician in the early assessment of children being evaluated for LNB as compared to the pleocytosis itself, as a single variable, can be discussed. It has previously been shown that pleocytosis with $\geq 90 \%$ mononuclear cells in CSF clearly discriminates Lyme meningitis from viral meningitis [19, 25-28]. However, there are patients without pleocytosis in CSF with cranial nerve palsy, fatigue and fever who will be detected by a positive NeBoP score and be recommended early start of antibiotic treatment. Furthermore, it has been shown that the majority of symptoms in children with LNB resolve within a few days after the start of antibiotic therapy [29], but whether an early start of treatment is favorable for long term clinical outcome is not clear [30].

A prediction model for LNB in children in a European setting has, to our knowledge, previously been presented only in one study [25]. However, children with acute facial nerve palsy without meningitis were not included, which qualifies our $\mathrm{NeBoP}$ score as a more relevant predictive test with a more adequate representation of all children being evaluated for LNB in a relevant European setting. Furthermore, a clinical prediction test would also be useful for adult LNB patients, but such a test has, to our knowledge, not yet been developed.

\section{Conclusion}

In conclusion, the overall diagnostic accuracy of the $\mathrm{NeBoP}$ score is high $(90 \%)$ and the test is suggested to be useful for decision-making about early antibiotic treatment in children being evaluated for LNB in European Lyme endemic areas.

\section{Additional file}

Additional file 1: Questionnaire. Study "Lyme Neuroborreliosis in children". (DOC $2013 \mathrm{~kb}$ )

\section{Abbreviations}

AUC: Area under the curve; Bb: Borrelia burgdorferi; Cl: Confidence interval; CSF: Cerebrospinal fluid; EM: Erythema migrans; IgG: Immunoglobulin G; IgM: Immunoglobulin M; LB: Lyme borreliosis; LNB: Lyme neuroborreliosis; LR: Likelihood ratio; NeBoP score: Neuroborreliosis prediction score; NPV: Negative predictive value; PCR: Polymerase chain reaction; PPV: Positive predictive value; ROC curve: Receiver operator characteristic curve.

\section{Competing interests}

The authors declare that they have no competing interests and none of the authors have any financial disclosure.

\section{Authors' contributions}

BHS planned study concept, design, organisation and realization of the study. BHS carried out data analysis, drafting of results and wrote the majority of the manuscript. JS and PEL contributed with critical revision of manuscript and important intellectual discussion of content. All authors have read and approved the final version of the manuscript.

\section{Acknowledgement}

Special thanks to the paediatricians MDs Catrin Furuhjelm, Maria Nordwall, Johan Anderzén, Michael Backhaus, Johan Mäkk and the staff at the paediatric clinics in Linköping, Norrköping, Jönköping, Skövde/Lidköping, Västerås and Falun for including patients in the study. Special thanks also to the paediatrician MD Sandra Andreasson at the paediatric clinic in Falun and to the medical student Mohammad Hammad at Linköping University for handling data and analyzing parts of the material. Excellent advice about statistical analyses was received from the statistician Jan Iver and excellent administrative support was received from research administrator Maria Pilawa-Podgurski, both at the Center for Clinical Research Dalarna. Funding was provided by the Center of Clinical Research Dalarna (CKF), the Swedish Society of Medicine, the Research Council in the Uppsala-Örebro region (RFR), The Samaritan Foundation and the Lions Foundation.

\section{Author details}

'Paediatric clinic, Falun General Hospital, Nissers väg 3, S-791 82 Falun, Sweden. ${ }^{2}$ Center for Clinical Research (CKF) Dalarna-Uppsala University, S-791 31 Falun, Sweden. ${ }^{3}$ Clinic of Infectious Diseases, Linköping University Hospital, S-581 85 Linköping, Sweden. ${ }^{4}$ Department of Clinical and Experimental Medicine, Medical Microbiology, Linköping University, S-581 85 Linköping, Sweden. ${ }^{5}$ Microbiological Laboratory, Medical Services, County Hospital Ryhov, S-551 85 Jönköping, Sweden.

Received: 25 June 2015 Accepted: 12 December 2015

Published online: 17 December 2015

\section{References}

1. Stanek G, O'Connell S, Cimmino M, Aberer E, Kristoferitsch W, Granstrom M, et al. European union concerted action on risk assessment in Lyme borreliosis: clinical case definitions for Lyme borreliosis. Wien Klin Wochenschr. 1996;108(23):741-7.

2. Steere AC. Lyme borreliosis in 2005, 30 years after initial observations in Lyme Connecticut. Wien Klin Wochenschr. 2006;118(21-22):625-33.

3. Strle F, Stanek G. Clinical manifestations and diagnosis of lyme borreliosis. Curr Probl Dermatol. 2009;37:51-110.

4. Bryant KA, Marshall GS. Clinical manifestations of tick-borne infections in children. Clin Diagn Lab Immunol. 2000;7(4):523-7.

5. Shapiro ED, Gerber MA. Lyme disease. Clin Infect Dis. 2000;31(2):533-42.

6. Mygland A, Ljostad U, Fingerle V, Rupprecht T, Schmutzhard E, Steiner I. EFNS guidelines on the diagnosis and management of European Lyme neuroborreliosis. Eur J Neurol. 2010;17(1):8-16. e11-14.

7. Avery RA, Frank G, Glutting JJ, Eppes SC. Prediction of Lyme meningitis in children from a Lyme disease-endemic region: a logistic-regression model using history, physical, and laboratory findings. Pediatrics. 2006;117(1):e1-7. 
8. Nigrovic LE, Thompson AD, Fine AM, Kimia A. Clinical predictors of Lyme disease among children with a peripheral facial palsy at an emergency department in a Lyme disease-endemic area. Pediatrics. 2008;122(5):e1080-5.

9. Garro AC, Rutman M, Simonsen K, Jaeger JL, Chapin K, Lockhart G Prospective validation of a clinical prediction model for Lyme meningitis in children. Pediatrics. 2009;123(5):e829-34.

10. Cohn KA, Thompson AD, Shah SS, Hines EM, Lyons TW, Welsh EJ, et al. Validation of a clinical prediction rule to distinguish Lyme meningitis from aseptic meningitis. Pediatrics. 2012;129(1):e46-53.

11. Tveitnes D, Oymar K, Natas O. Acute facial nerve palsy in children: how often is it lyme borreliosis? Scand J Infect Dis. 2007;39(5):425-31.

12. Skogman BH, Croner S, Nordwall M, Eknefelt M, Ernerudh J, Forsberg P. Lyme neuroborreliosis in children: a prospective study of clinical features, prognosis, and outcome. Pediatr Infect Dis J. 2008;27(12):1089-94.

13. Broekhuiisen-van Henten DM, Braun KP, Wolfs TF. Clinical presentation of childhood neuroborreliosis; neurological examination may be normal. Arch Dis Child. 2010;95(11):910-4.

14. Christen HJ. Lyme neuroborreliosis in children. Ann Med. 1996;28(3):235-40.

15. Oymar K, Tveitnes D. Clinical characteristics of childhood Lyme neuroborreliosis in an endemic area of northern Europe. Scand J Infect Dis. 2009;41(2):88-94

16. Christen HJ, Hanefeld F, Eiffert $H$, Thomssen R. Epidemiology and clinical manifestations of Lyme borreliosis in childhood. A prospective multicentre study with special regard to neuroborreliosis. Acta Paediatr Suppl. 1993;386: $1-75$.

17. Sood SK. What we have learned about Lyme borreliosis from studies in children. Wien Klin Wochenschr. 2006;118(21-22):638-42.

18. Belman AL, lyer M, Coyle PK, Dattwyler R. Neurologic manifestations in children with North American Lyme disease. Neurology. 1993;43(12):2609-14.

19. Shah SS, Zaoutis TE, Turnquist J, Hodinka RL, Coffin SE. Early differentiation of Lyme from enteroviral meningitis. Pediatr Infect Dis J. 2005;24(6):542-5.

20. Tuerlinckx D, Bodart E, Garrino MG, de Bilderling G. Clinical data and cerebrospinal fluid findings in Lyme meningitis versus aseptic meningitis. Eur J Pediatr. 2003;162(3):150-3.

21. Bremell D, Mattsson N, Wallin F, Henriksson J, Wall M, Blennow K, et al. Automated cerebrospinal fluid cell count-new reference ranges and evaluation of its clinical use in central nervous system infections. Clin Biochem. 2014;47(1-2):25-30.

22. Hansen K, Lebech AM. Lyme neuroborreliosis: a new sensitive diagnostic assay for intrathecal synthesis of Borrelia burgdorferi-specific immunoglobulin G, A, and M. Ann Neurol. 1991;30(2):197-205.

23. Baumann M, Birnbacher R, Koch J, Strobl R, Rostasy K. Uncommon manifestations of neuroborreliosis in children. Eur J Paediatr Neurol. 2010; 14(3):274-7.

24. Arnez M, Pleterski-Rigler D, Luznik-Bufon T, Ruzic-Sabljic E, Strle F. Children with multiple erythema migrans: are there any pre-treatment symptoms and/or signs suggestive for central nervous system involvement? Wien Klin Wochenschr. 2002;114(13-14):524-9.

25. Tuerlinckx D, Bodart E, Jamart J, Glupczynski Y. Prediction of Lyme meningitis based on a logistic regression model using clinical and cerebrospinal fluid analysis: a European study. Pediatr Infect Dis J. 2009; 28(5):394-7.

26. Waespe N, Steffen I, Heininger U. Etiology of aseptic meningitis, peripheral facial nerve palsy, and a combination of both in children. Pediatr Infect Dis J. 2010;29(5):453-6.

27. Eppes SC, Nelson DK, Lewis LL, Klein JD. Characterization of Lyme meningitis and comparison with viral meningitis in children. Pediatrics. 1999;103(5 Pt 1):957-60.

28. Tveitnes D, Natas OB, Skadberg O, Oymar K. Lyme meningitis, the major cause of childhood meningitis in an endemic area: a population based study. Arch Dis Child. 2012;97(3):215-20.

29. Thorstrand C, Belfrage E, Bennet R, Malmborg P, Eriksson M. Successful treatment of neuroborreliosis with ten day regimens. Pediatr Infect Dis J. 2002;21(12):1142-5.

30. Skogman BH, Glimaker K, Nordwall M, Vrethem M, Odkvist L, Forsberg P. Long-term clinical outcome after Lyme neuroborreliosis in childhood. Pediatrics. 2012;130(2):262-9.

\section{Submit your next manuscript to BioMed Central and we will help you at every step:}

- We accept pre-submission inquiries

- Our selector tool helps you to find the most relevant journal

- We provide round the clock customer support

- Convenient online submission

- Thorough peer review

- Inclusion in PubMed and all major indexing services

- Maximum visibility for your research

Submit your manuscript at www.biomedcentral.com/submit 